\title{
GLOBAL DATA SPATIALLY INTERRELATE SYSTEM FOR SCIENTIFIC BIG DATA SPATIAL-SEAMLESS SHARING
}

\author{
Jieqing Yu ${ }^{\mathrm{a}}$, Lixin $\mathrm{WU}^{\mathrm{b}, \mathrm{a}, \mathrm{c}^{*}}$, Yizhou Yang ${ }^{\mathrm{c}}$, Xie Lei ${ }^{\mathrm{d}}$, Wang He ${ }^{\mathrm{d}}$
}

\author{
${ }^{a}$ School of Environment Science and Spatial Informatics, China University of Mining and Technology, Xuzhou, China \\ (yujieqing@gmail.com) \\ ${ }^{\mathrm{b}}$ IoT (Mine Perception) Centre, China University of Mining and Technology, Xuzhou, China; awulixin@ 263.net \\ ${ }^{\mathrm{c}}$ Institute of Geo-informatics and Digital Mine Research, Northeastern University, Shenyang, China \\ ${ }^{\mathrm{d}}$ College of Geoscience and Surveying Engineering, China University of Mining and Technology (Beijing), Beijing, \\ China
}

Commission: IV

KEY WORDS: data sharing, global data spatially interrelate system, earth system spatial grid, SDOG-ESSG, big data management

\begin{abstract}
:
A good data sharing system with spatial-seamless services will prevent the scientists from tedious, boring, and time consuming work of spatial transformation, and hence encourage the usage of the scientific data, and increase the scientific innovation. Having been adopted as the framework of Earth datasets by Group on Earth Observation (GEO), Earth System Spatial Grid (ESSG) is potential to be the spatial reference of the Earth datasets. Based on the implementation of ESSG, SDOG-ESSG, a data sharing system named global data spatially interrelate system (GASE) was design to make the data sharing spatial-seamless. The architecture of GASE was introduced. The implementation of the two key components, V-Pools, and interrelating engine, and the prototype is presented. Any dataset is firstly resampled into SDOG-ESSG, and is divided into small blocks, and then are mapped into hierarchical system of the distributed file system in V-Pools, which together makes the data serving at a uniform spatial reference and at a high efficiency. Besides, the datasets from different data centres are interrelated by the interrelating engine at the uniform spatial reference of SDOGESSG, which enables the system to sharing the open datasets in the internet spatial-seamless.
\end{abstract}

\section{INTRODUCTION}

Most of scientific researches rely on scientific data, especially, the Earth System Science (ESS). As the development of Earth observation technology, more and more scientific data are obtained for scientific research. However, without a data sharing system, these data are nothing. As Carlson ${ }^{[1]}$ said, a perfect data-sharing system is science's unobtainium.

A variety of data centres and data sharing platforms, have been built in the past decades, e.g., NASA Earth Observing System Data and Information System (EOSDIS) ${ }^{[2]}$, USGS Earth Resources Observation and Science Centre (EROS) ${ }^{[3]}$, NOAA The Comprehensive Large Array-data Stewardship System (CLASS) ${ }^{[4]}$, Infrastructure for Spatial Information in Europe (INSPIRE) ${ }^{[5]}$, World Data System (WDS) ${ }^{[6]}$, Global Earth Observation System of Systems (GEOSS) ${ }^{[7]}$, Google earth, and Tianditu ${ }^{[8]}$. NASA EOSDIS, USGS EROS, and NOAA CLASS are the three most frequently used data resources for earth scientists around the world perhaps. All of them are very big data centres that distribute a vast of earth observation data to the public. WDS is a new version of world data centre under The International Council for Science (ICSU). It collects datasets from its member organizations based on the so called data policy ${ }^{[9]}$, and offers a uniform portal to access the data from different members through the internationally recognized standards and the common infrastructure built by the members. INSPIRE is a project founded by European Union (EU) in 2007 to build an infrastructure to share the environmental spatial information across Europe based on OGC standards. Google earth is software platform developed by Google ${ }^{\mathrm{TM}}$ Corporation to sharing global image online. Tianditu is an online geographic information sharing platform developed by National Administration of Surveying, Mapping and Geo-information of China.

As seen, the concerning of the data centres and data sharing platforms are the data sharing policies, common infrastructure, uniform portal, and online data services. Few of them pay much attention to a uniform spatial reference. As known, there are hundreds of spatial references, e.g., UTM, Gauss-Kruger, and WGS-84, in use simultaneously for different datasets. Scientists have to download a set of datasets from data centres, and then make many spatial transformations on the datasets for further data analysis manually in order to make a research. However, the spatial transformation is tedious, boring, and time consuming. It will sometime be a problem for non-specialist in GIS, which may discourage the users from the usage of the data, and hence reduce the scientific innovation. It will obviously benefit the scientists a lot if a spatial-seamless data sharing system with no spatial transformation is needed.

Earth System Spatial Grid (ESSG) proposed by Wu and Yu ${ }^{[10]}$ has recently been established as the framework of Earth datasets by $\mathrm{GEO}^{[11]}$, who is a governmental and international level organization intending to build a Global Earth Observation System of Systems (GEOSS) to share and interrelate the worldwide datasets freely and globally. Thus, ESSG could be the uniform spatial reference for data sharing in GEOSS. Based on ESSG, Wu ${ }^{[12]}$ proposed a new mode of data sharing, named Global Data Spatial Interrelate (GASE), for global scientific data sharing. The GASE is a typical spatial-seamless data sharing system. This paper will make an detail introduction to GASE, including the architecture and implementation of the GASE. 


\section{ARCHITECTURE OF GLOBAL DATA SPATIALLY INTERRELATE SYSTEM}

\subsection{EARTH SYSTEM SPATIAL GRID FOR SCIENTIFIC DATA REPRESENTATION}

Earth System Spatial Grid (ESSG) is an exhaustive coverage, hierarchical structure, approximate sized, definite frame, unique coding and multiple granularities three-dimensional (3D) global grid system intended for Earth System Science research. Yu ${ }^{[13,14]}$ had given out an implementation of ESSG, named SDOG-ESSG, based on Spheroid degenerated-octree grid with refinement on radius. It is achieved by a spheroid degenerated-octree subdivision on a large reference spheroid whose radius is the twice of the average Earth radius, and then by a radial bisection on the produced grids. A coupled degenerated-Z curve (CDZ-curve) was used to fill the SDOG-ESSG to get a unique code for each grid ${ }^{[13,14]}$. The large reference spheroid, the features of exhaustive coverage, hierarchical structure, approximate sized, multiple granularities, and the unique code make the SDOG-ESSG being an effective spatial reference for Earth datasets and hence being a suggested reference for scientific data exchange by GEO. Fig. 1 shows an example of SDOG-ESSG and its CDZ-curve.
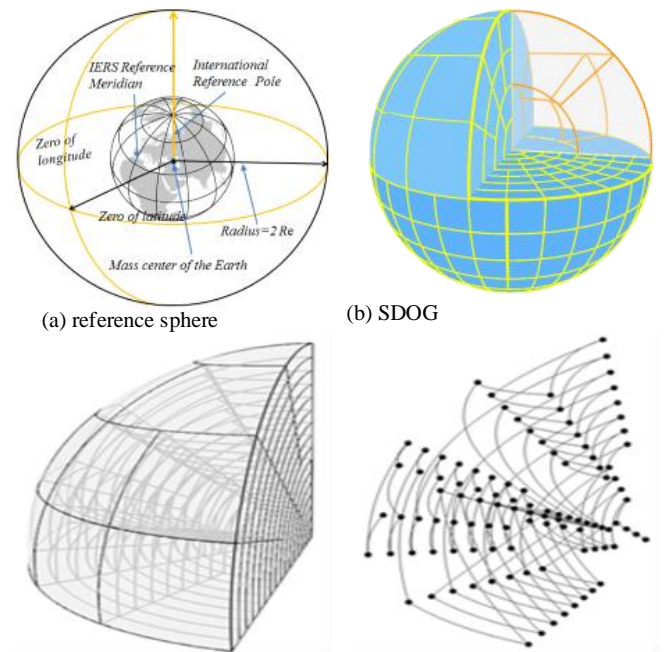

(b) SDOG

(c) SDOG-ESSC

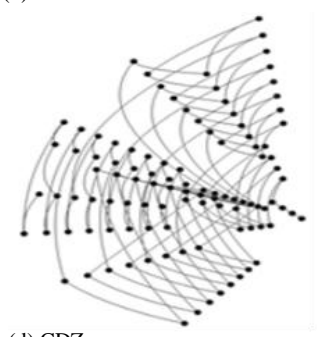

(d) CDZ-curve

Fig.1 SDOG-based Earth System Spatial Grid (SDOG-ESSG) and its CDZ-curve.

The grid element in SDOG-ESSG is not only a 3D voxel in the space of planet Earth, but also a 3D container for the spatial data ${ }^{[12]}$. Any dataset in the space can be considered as information that is attached to the grid. From the method of SDOG-ESSG encoding, the information of location, shape and granularity for a grid is embodied in the code. In other words, the grid code contains all the spatial information needed. Thus, a triple (C, T, A) model ${ }^{[15]}$ is enough to represent the temporal-spatial objects (phenomenon) based on SDOG-ESSG (Fig. 2). In the triple model, C refers to the grid code of SDOG-ESSG, T refers to the time stamp for the temporal datasets, and A is the attribute values of the temporal-spatial objects (phenomenon). Referring to the resolutions of datasets and application demands, any dataset can be resampled or re-gridded into SDOG-ESSG at a certain granularity level, so as to get the value of spatial $C$ and attribute $A$ for the elements of triple model ${ }^{[12]}$.

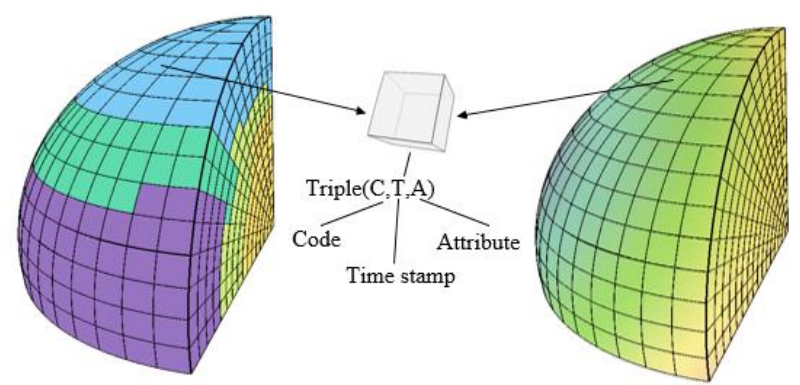

Fig. 2 Data representation in SDOG-ESSG

\subsection{GLOBAL DATA SPATIALLY INTERRELATE SYSTEM BASED ON SDOG-ESSG}

Based on SDOG-ESSG, a Global Data Spatially Interrelate System (GASE) for global datasets spatial-seamless sharing is proposed by $\mathrm{Wu}{ }^{[12]}$. GASE mainly consists of V-Pools, ESSG engine, interrelating engine, and tools engine (Fig. 3). V-Pools is a large distributive database system that keeps a vast of datasets for GASE. All datasets are transformed into SDOG-ESSG triples before being flushed into the V-Pools. The interrelating engine interrelates the various datasets from the data centers seamless in a unified spatial reference. It provides remote scientific data finding, and spatial transformation services to the ESSG engine if the dataset required is not found in the V-Pools. ESSG engine gets data either from interrelating engine or from V-Pools depending on whether the data is in the V-Pools, and then provides data services to the data users or tools engine. The tools engine provides online analysis service to the data users, such as online visualization, if further analysis is required. Among these components, V-Pools is the key component to manage the dataset in a uniform spatial reference and to decide the performance of data service, while interrelating engine is key component that makes the data service spatial-seamless and provide a variety of datasets to the users. Both of them are vital to the GASE. We will then discuss them in detail in the following.

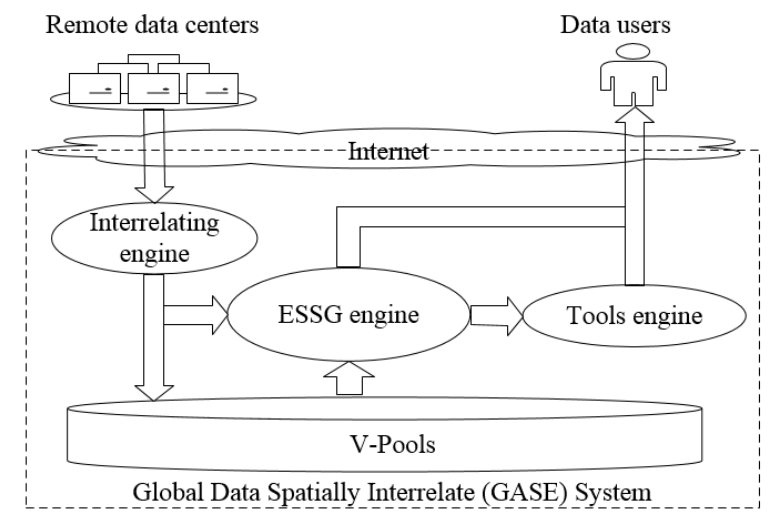

Fig.3 Architecture of GASE. GASE is mainly composed of V-Pools, ESSG engine, interrelating engine and tools engine. V-Pools is a data archiving system that manages a vast of datasets. ESSG engine is data providing system to the tools engine and the data users. Tools engine is a collection of tool sets that provide further process service on the retrieval data to the users. Interrelating engine is a data finding, updating and automatically spatial transforming system that gives supplements to the V-Pools. 


\section{MANAGEMENT OF BIG DATA IN THE V-POOLS}

Any dataset is resampled into SDOG-ESSG before being flushed into the V-Pools. The methods of resampling dataset into SDOG-ESSG is presented in [14, 15]. The biggest problem for data management in V-Pools is the data volume. The data volume in the $\mathrm{V}$-pools will be very huge and hence be far beyond the ability of a single data server. Advance storage technologies, such as distributive storage and cloudbased storage, is required to manage the big data. Fortunately, we can take full advantages of SDOG-ESSG to build a scalable, distributive or even cloud-based big data managing system. The main idea of managing the big data in V-Pools based SDOG-ESSG is to build a hierarchical system for the big data and then to divide each dataset into blocks so as to take full ability of the IOs.

\subsection{HIERARCHICAL SYSTEM AND DATA PARTITION IN V-POOLS}

As known from the method of grid subdivision, SDOG-ESSG is a hierarchical grid system with each grid encoded uniquely, which makes itself extremely suitable for big data management in distributive environment. In SDOG-ESSG, grids of low level can be grouped into a grid of high level, and grids of high level can be grouped into a grid of higher level, and so forth. Thus, a hierarchical tree can be built according to the grouping strategy. In the hierarchical tree, grids of different level are located on the nodes of different depth. Each grid owns one single parent and a number of children, and can be labeled by a unique code that indicates the extent, granularity and location of the grid. The extent of the grid could then be used for the indexing of a block of voxel data, such as triples.

As known, any dataset has a limitation on the resolution. So, according to the resolution we can attach any dataset to the corresponding depth of the hierarchical tree in which the granularity of the grid is no smaller than the resolution of the dataset. For example, datasets with resolution at $1 \mathrm{Km} \times 1 \mathrm{Km}$ can be attached to the node grid at level 6 whose granularity is about $50 \mathrm{Km} \times 50 \mathrm{Km}$.

Fig. 4 gives an illustration on the hierarchical tree for big data management. The whole tree is composed of a vast of nodes of different depths. The root of the hierarchical tree is set to be the globe. The grid of SDOG-ESSG at level $k \bullet d$ is mapped into the node at depth $k$, where $d$ is a number no smaller than 1 and refers to the difference of grid level between nodes of two adjacent depth. Each node grid is given a code indicated by $\mathrm{C}^{n}$, where $n$ is the level of the node grid. Dataset is firstly resampled or re-grid into SDOG-ESSG at the level corresponding to its resolution, and then is divided into small blocks according to the extent of the node grid that it is going to be attached to. The level of the node grid $\left(n_{d}\right)$ can be jointly decided by the resampling level $\left(n_{r e}\right)$, the minimum difference of level $\left(D_{\min }\right)$ and maximum difference of level $\left(D_{\max }\right)$ between the resampled datasets and the node grid. The relation is shown in the following,

$$
n_{r e}+D_{\text {min }}<n_{d} \leq n_{r e}+D_{\text {max }}, \quad \text { Formula } 1
$$

where, $D_{\max }-D_{\min }=d-1$.

The way of attaching the block to the node-grid is to take the code of node grid as the main part of the block code. The code of block consists of theme code, node grid code, and time stamp. Theme code (A) refers to the identification of the dataset. The theme code should be unique in the GASE system, e.g., the attribute classification code based on the industry standards. Time stamp (T) is a string that records the date or the time when the dataset is produced or observed in the forms of 'yyyymmddhh', such as '20000101' and '2000010110'. The three components are joined together by a separator character like '_' in sequence. For example, ' Temp01_10265221_20000101' refers to the dataset of 'Temp01' produced on January 1, 2000 with its spatial extent within the boundary of grid code '10265221'.

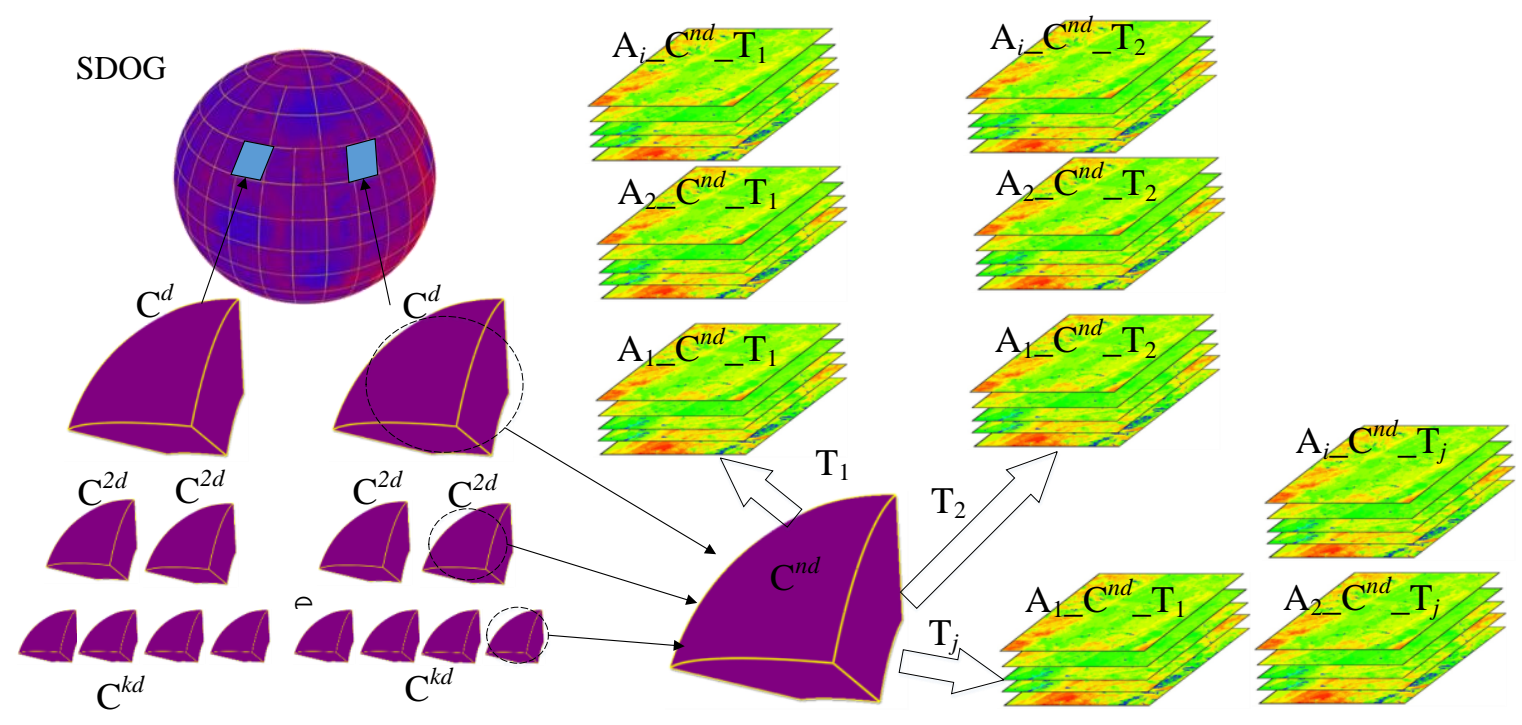

Fig.4 Hierarchical system for big data management based on SDOG-ESSG. The globe is divided into grids of different level by the method of degenerated octree subdivision. Grids of the same level consist of the nodes of the same depth of the hierarchical tree. On the other hand, datasets are divided into small blocks by the method of SDOG-ESSG subdivision. Each block is attached to the corresponding node grid, and jointly coded by dataset identification (A), node gird code (C) and time stamp (T) separated by a special character like '.'. The hierarchical system is then mapped into the file system or the distributed file system. 


\subsection{DATA STORAGE IN THE V-POOLS}

The hierarchical tree is a logical way of managing the big data based on SDOG-ESSG, and has to be mapped into the database. Here, a mixed way of relation database and file system is used to store the big data in V-Pools. The relation database is used to store the metadata, e.g., the difference of grid level $(d)$ between nodes of two adjacent depth, the minimum difference of level $\left(D_{\min }\right)$ and maximum difference of level $\left(D_{\max }\right)$ between the resampled datasets and the node grid, resampling level of each dataset, boundary box of each dataset, and any other useful metadata of each dataset. The file system is used to store the block data and its hierarchical index. Each block data is written into a file that records the value of each grid in sequence. The key problem of the storage is to build a hierarchical index for the block data. However, the hierarchical index, which acts as a hierarchical folder system, can be made by mapping the hierarchical tree into the file system. In the hierarchical folder system, each folder refers to a node of the hierarchical tree, and is named by the grid code. The block data attached to node grid is put into the folder referring to the node. For example, the folder ' 10265221 ' is attached to the node grid ' 10265221 ', and may contain both sub-folder '1026522122' and data block '1026522122', where '1026522122' is the father of the father grid of '10265221'. Thus, the way to find the data block is to construct a full path from the root of the hierarchical tree to the attached node grid, e. g.,

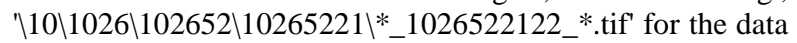
block '140158034'.

This hierarchical folder system can be easily extended to cluster. As known, the efficiency of data accessing is a key measurement to evaluate the performance of a big data management system. However, the bottleneck always lies on the data accessing on IOs. An efficient way is to make the data accessing taken from different disks at the same time. Considering that the data accessed at a time is always spatially adjacent, the data nearby needs to be flushed into different disks so as to explore the full ability of IO. As argued above, any dataset is divided into small blocks. If these blocks are distributed into different disks, the ability of disks can be explored fully. According to coding system of SDOG-ESSG, the code of the $d^{\text {th }}$ level child of a grid owns a suffix of $d$-digit ranging from 0 to $8^{d}-1$. A good balance on the data distribution will be achieved, if the blocks are assigned into numbered disks according to the last $d$ digitals of the code. For example, data block with a code ended by a suffix of '15' is attached to the disk '15'. Thus, most of the disks will work at the same time, which will be no doubt to enhance the ability of data accessing, if a large extent of data is requested.

Even though, there is a limitation on the capacity of a single cluster. A much larger distribution system which is open and scaleable is required for V-Pools. Fig. 5 give an illustration on the architecture of V-Pools. It is composed of various data houses that are interconnected to by Internet. Each data house may either be a cluster, or a voluntary storage node contributed by any institute. It is responsible for several datasets independently. If it is a cluster, the datasets will be distributed uniformly on the storage nodes following the strategy of blocks partitioning and the assignment of round robin argued above; otherwise, the whole dataset will be flushed into a single node after blocks partitioning. Therefore, the full path for the data block '1026522122' may be 'A2\Node22\10\1026\102652\10265221\*_1026522122_*.tif', where the paths of data house and storage nodes are included.

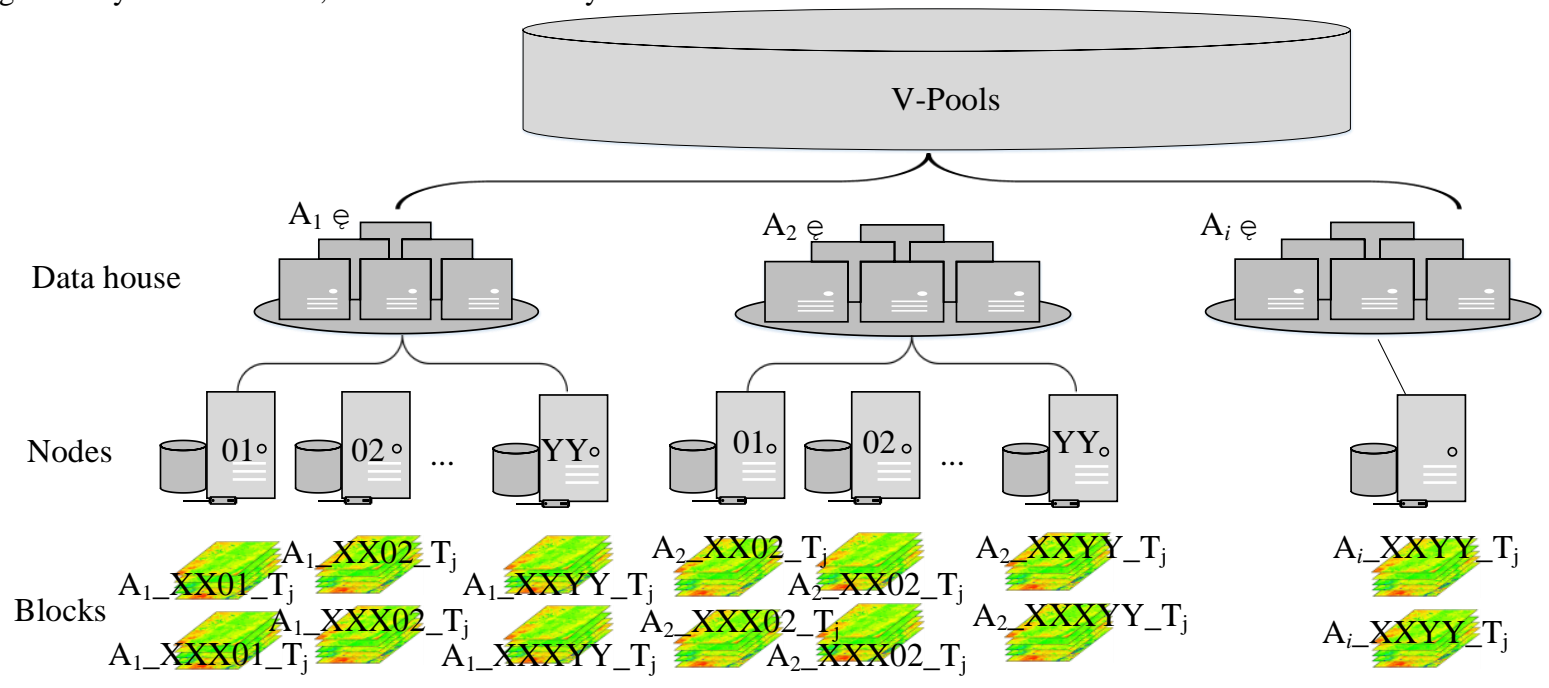

Fig. 5 V-Pools is composed of many data house, each of which may either be a cluster or a single node on the Internet, and is responsible for several dataset independently. The datasets are divided into blocks which are mapped into the storage nodes according to round robin strategy.

\subsection{DATA RETRIEVAL IN THE V-POOLS}

The only thing that the users need to do to get the desirable data from this system is to submit a request that contains the information of dataset descriptions, time range, resolution, and spatial extent to the system. The system will handle it automatically, and then return the exact datasets or the production in a unified spatial reference. No more attention is need to be paid on the spatial transformation, which will save the data users a lot of time and frees the data users from boring work in spatial transformation.

Dataset descriptions are used to determinate the identification of the dataset that the user requests. The work is usually done by ESSG engine. Time range contains the information of time span that the user concerns. It is transformed into two time stamps in the ESSG engine in advance. Resolution is the key information to decide the resampling level of the dataset requested, and the level of node grid that the dataset attached 
to. Spatial extent is divided into small grids at the node level. Once the node grids and their codes are obtained, a full path that indicates the location of the block data can be generated for further retrieving by the identification of the dataset, the node grid codes and their father grids' codes. The work of searching the exact data file in the full path can be done by the searching expressions which are generated in advance in the memory to avoid extra searching in the file system. The expression is a mathematical expression that defines the range of block code using the node-grid codes, theme code and two time stamp, such as '> Temp01_140158034_20000101 and < Temp01_140158034_20000201', and '> Temp01_140158034_20000101 and < Temp01_140158034_20000201'. The final result can be gotten by the execution of the searching expressions in the full path.

\section{INTERRELATING ENGINE}

Any data center has a limitation on its data resource. There is is a huge data resource in the internet where everybody can contribute their datasets. If a data sharing platform can interrelate their system to the open data resource in the internet, it will no doubt be more attractive. So far, a lot of data sharing system has been developed to try to provide data service upon multiply data centres organizations in the internet, such GEOSS, INSPIRE, and WDS. However, what they do is to interrelate datasets from different data centres through a uniform data sharing policy, a well-recognized international standard, a common infrastructure and a uniform portal. Little attention has been paid to interrelate the datasets in a uniform spatial reference.

Referring to above problem, a component of spatial data interrelating, named interrelating engine, is designed to interrelate the datasets from different data centres in a uniform spatial reference in GASE. It is composed of three components (Fig. 6), including the components of updating, searching and conversion. Updating component is responsible for updating the V-Pools data from remote data centres in case of any dataset is updated. It is a little similar to software updating, who queries the server periodically and then updates itself when there is a new version available. The difference lies that the focus of the software updates is software itself while that of the updating component in interrelating engine is open datasets. The updating component queries the data centre periodically to test whether there is a new version of the dataset. If a new version is found, the component will download the data automatically, and then summit the new version dataset to conversion component. The conversion component will resample the datasets of various spatial references into a uniform spatial reference, which is SDOG-ESSG, and then divide them into blocks which are to be stored in V-Pools. Searching component is a component to discover and download new dataset from the internet through standard protocols, such as OGC catalog service-web (CSW) and web coverage service (WCS). The dataset downloaded is also submitted to conversion components, and then return the resampled data blocks to ESSG engine and V-Pools at the same time.

\section{A PROTOTYPE FOR GASE}

A prototype for GASE has been developed with $\mathrm{C}++$ and Java language, Qt, GDAL open source library. It was built upon a six-node cluster (Fig.7), where four of them were used for storage nodes (V-Pools), one for ESSG server and the rest one for interrelating engine. The six nodes were all connected by a router which was connect to the Internet. We took the datasets of global $30 \mathrm{~m} \mathrm{DEM}$ as experiment data to implement the V-Pools and ESSG server. $D_{\min }, D_{\max }$ and $d$ were set to 5, 7 and 3, respectively. The volume for global 30 DEM is about 500TB. It was resampled into SDOG-ESSG at the level of 19 , and divided into blocks at the level of 12 . Block with node codes ended by ' 0 ' and ' 4 ' were stored in node ' 0 ' and ' 4 ', and that ended by ' 1 ' and ' 5 ' were stored in node 1 , and so on. Our test showed that the retrieval of data is convenient and high efficient. The retrieval of Xuzhou region DEM is near real time. Fig. 8 shows the interface of GASE and the return DEM of Xuzhou region.

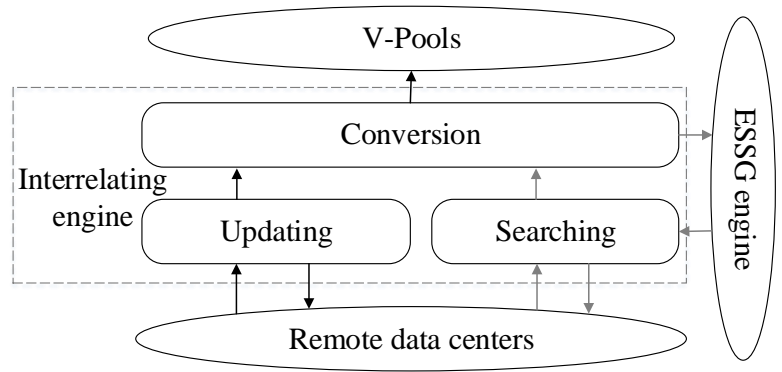

Fig.6 Architecture of interrelating engine. It is mainly composed of updating, searching and conversion components.

NASA developed a language of IIMSAQL to help the users to obtain data from its EOSDIS system. Thus, based on IIMSAQL we implemented an interrelating engine, by which datasets from NASA EOSDIS can be obtained and automatically updated in the form of the uniform spatial reference of SDOG-ESSG. Fig. 9 shows the interface of interrelating engine which updates the datasets 'ADVANCED MICROWAVE SOUNDING UN17-A (AMSU-A) SWATH FROM NOAA-17' into V-Pools every day automatically in the spatial reference of SDOG-ESSG.

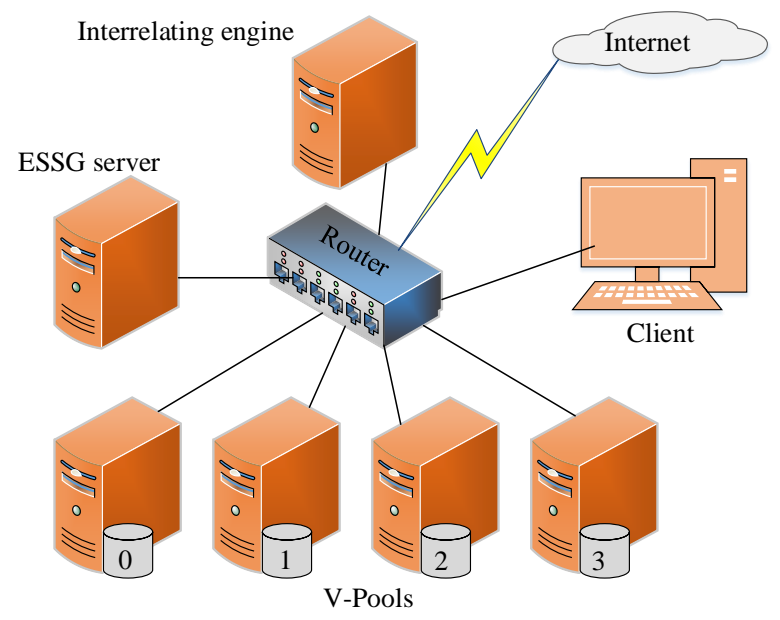

Fig.7 The environments of the GASE prototype.

\section{SUMMARIES AND REMARKS}

Data sharing is vital to earth science research. However, a good data sharing system with spatial-seamless services is also very important to scientists, which will prevent the scientists from tedious, boring, and time consuming work of spatial transformation, and hence encourage the usage of the scientific data, and increase the scientific innovation. Having 
been adopted as the framework of Earth datasets by GEO, which is a governmental and international level organization intending to share the worldwide datasets freely and globally, ESSG is potential to be the spatial reference of the Earth datasets. Based on the implementation of ESSG, named SDOG-ESSG, a data sharing system named global data spatially interrelate system (GASE) was design to make the data sharing spatial-seamless.

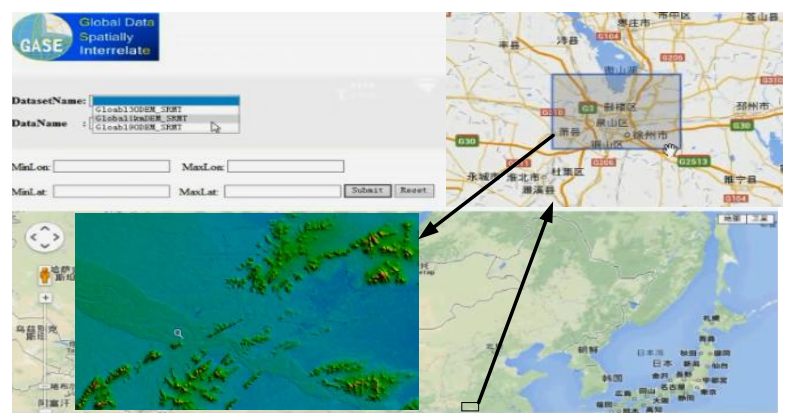

Fig.8 Retrieving DEM in Xuzhou region from GASE.

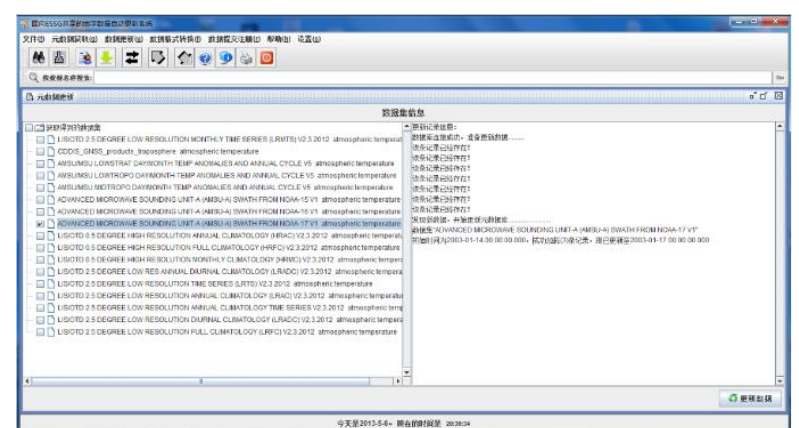

Fig.9 Updating of the dataset 'ADVANCED MICROWAVE SOUNDING UN17-A (AMSU-A) SWATH FROM NOAA17 ' into V-Pools automatically in the spatial reference of SDOG-ESSG.

The system was composed of V-Pools, ESSG server, interrelating engine and tools engine, among which V-Pools and interrelating engine are the most important two in the system. The V-Pools is responsible for the big data management at the uniform spatial reference of SDOG-ESSG, and offers a high performance accessing to the datasets. The dataset is firstly resampled into SDOG-ESSG, and is divided into small blocks, and then are mapped into hierarchical system of the distributed file system, which together makes the data serving at a uniform spatial reference and at a high efficiency. The interrelating engine interrelated the datasets from various data centres at the uniform spatial reference of SDOG-ESSG, and provides data updating and downloading from the data centres automatically. The component enable the system to sharing the open datasets in the internet spatialseamless.

\section{ACKNOWLEDGEMENT}

This study is funded by National Natural Science Foundation of China (No. 41301432; 40930104), and the Fundamental Research Funds for the Central Universities (No. 2013QNB10).

\section{REFERENCE}

1. Carlson D., A lesson in sharing [J]. Nature, 2011, 469:293.

2. NASA EOSDIS http://reverb.echo.nasa.gov/reverb/, [2014-3-31]

3. USGS EROS [EB/OL], https://eros.usgs.gov, [2014-331].

4. NOAA CLASS [EB/OL], www.class.noaa.gov, [2014-331].

5. INSPIRE [EB/OL], http://inspire.ec.europa.eu/, [2014-331].

6. WDS [EB/OL], http://www.icsu-wds.org/, [2014-3-31].

7. GEOSS [EB/OL], http://www.earthobservations.org/geoss.shtml, [2014-331].

8. Tianditu http://en.tianditu.com/map/index.html, [2014-3-31].

9. ICSU, WDS Data Policy [EB/OL]. http://www.icsuwds.org/services/WDS_Data_Policy.pdf, [2014-3-31]

10. Wu Lixin, Yu Jieqing. Earth System Spatial Grid and Its Application Mode [J]. Geography and Geo-information Science, 2012, 28(01): 7-13.

11. GEO, GEO WORK PLAN 2012-2015[EB/OL], http://www.earthobservations.org/docshow.php?id=129, [2014-3-31].

12. Lixin WU, Jieqing Yu, Yizhou Yang, et al. Spatial Big Data Organization, Access and Visualization with ESSG [C]. International Archives of the Photogrammetry, Remote Sensing and Spatial Information Sciences. 2013, XL-4/W2: 51-56.

13. Yu Jieqing, Wu Lixin. Adaptable Spheroid DegeneratedOctree Grid and Its Coding Method [J]. Geography and Geo-Information Science, 2012, 28(1):14-18.

14. Yu Jieqing. SDOG-based Earth System Spatial Grid and Its Application and Three Dimensional Modeling [D]. Beijing Normal University, 2012.

15. Yu Jieqing, $\mathrm{Wu}$ Lixin, Zi Guojie, et al. SDOG-based multi-scale 3D modeling and visualization on global lithosphere[J]. Sci China Earth Sci, 2012, 55(6): 10121020. 